\title{
The need for quality assurance of health apps
}

\author{
Brian McMillan National Institute for Health Research academic clinical fellow in primary care ${ }^{1}$, \\ Eamonn Hickey medical student ${ }^{1}$, Caroline Mitchell senior clinical lecturer ${ }^{1}$, Mahendra Patel senior \\ lecturer $^{2}$
}

${ }^{1}$ Academic Unit of Primary Medical Care, Northern General Hospital, Sheffield S5 7AU, UK; ${ }^{2}$ Department of Pharmacy, University of Huddersfield, Huddersfield, UK

With patients and healthcare professionals increasingly using health apps, Armstrong's article is timely. ${ }^{1}$ At the Academic Unit of Primary Medical Care in Sheffield we examined quality assurance for health behaviour change apps by adapting the 2014 NICE behaviour change guidance. ${ }^{2}$ We identified nine areas that are important to consider when examining the quality of such apps-purpose, planning and development, usability, assessment and tailoring, behaviour change technique, maintenance and relapse, evaluation, documentation, and data protection. ${ }^{3}$ Behaviour change apps from the now closed NHS apps library were clear in terms of purpose, but few showed evidence of thorough planning and development or of addressing how behavioural changes could be maintained. There was little focus on usability, and evidence for the evaluation of app efficacy was poor. Documentation varied, and only a minority showed evidence of initial assessment and tailoring.

Although the quality of apps varies greatly, the RCP's advice to avoid apps that lack a CE mark seems extreme. Until a clear quality assurance scheme is widely adopted, it seems sensible to view apps with the same degree of healthy scepticism that we afford other online sources of information. We look forward to a time when less vigilance is needed and there is a process in place to reassure healthcare professionals that the apps that they are using or recommending are reliable and trustworthy. It will be interesting to see how well the proposed National Information Board four step assessment process deals with these problems.

Competing interests: None declared.

Full response at: www.bmj.com/content/351/bmj.h4597/rr.

Armstrong S. Which app should I use? BMJ 2015;351:h4597. (9 September.) 2 National Institute for Health and Care Excellence. Behaviour change: individual approaches. 2014. www.nice.org.uk/guidance/ph49.

McMillan B, Hickey E, Patel MG, et al. Quality assessment of a sample of mobile app-based health behavior change interventions using a tool based on the National Institute of Health and Care Excellence behaviour change guidance. Patient Educ Counsel (forthcoming).

Cite this as: BMJ 2015;351:h5915

๑ BMJ Publishing Group Ltd 2015 